Reactive Rayleigh-Taylor systems: Front propagation and non-stationarity

This article has been downloaded from IOPscience. Please scroll down to see the full text article.

2011 EPL 9454004

(http://iopscience.iop.org/0295-5075/94/5/54004)

View the table of contents for this issue, or go to the journal homepage for more

Download details:

IP Address: 141.34.3.98

The article was downloaded on 03/01/2012 at 15:36

Please note that terms and conditions apply. 


\title{
Reactive Rayleigh-Taylor systems: Front propagation and non-stationarity
}

\author{
L. Biferale ${ }^{1,4,7}$, F. Mantovani ${ }^{2}$, M. Sbragaglia ${ }^{1}$, A. Scagliarini ${ }^{1,3(a)}$, F. Toschi ${ }^{4,5,7}$ and R. Tripiccione $^{6}$ \\ ${ }^{1}$ Department of Physics and INFN, University of Tor Vergata - Via della Ricerca Scientifica 1, \\ I-00133 Rome, Italy, EU \\ ${ }^{2}$ Deutsches Elektronen Synchrotron (DESY) - D-15738 Zeuthen, Germany, EU \\ ${ }^{3}$ Department of Fundamental Physics, University of Barcelona - Carrer de Martí i Franquès 1, \\ 08028 Barcelona, Spain, EU \\ ${ }^{4}$ Department of Applied Physics and Department of Mathematics and Computer Science, \\ Eindhoven University of Technology - 5600 MB Eindhoven, The Netherlands, EU \\ ${ }^{5}$ CNR-IAC - Via dei Taurini 19, I-00185 Rome, Italy, EU \\ ${ }^{6}$ Department of Physics and INFN, University of Ferrara - I-44100 Ferrara, Italy, EU \\ ${ }^{7}$ International Collaboration for Turbulence Research (ICTR)
}

received 24 January 2011; accepted in final form 24 April 2011

published online 27 May 2011

PACS 47.20.Ma - Interfacial instabilities (e.g., Rayleigh-Taylor)

PACS 47.70. $-\mathrm{n}$ - Reactive and radiative flows

PACS $47.45 . \mathrm{Ab}-$ Kinetic theory of gases

\begin{abstract}
Reactive Rayleigh-Taylor systems are characterized by the competition between the growth of the instability and the rate of reaction between cold (heavy) and hot (light) phases. We present results from state-of-the-art numerical simulations performed at high resolution in $2 \mathrm{~d}$ by means of a self-consistent lattice Boltzmann (LB) method which evolves the coupled momentum and temperature equations and includes a reactive term. We tune parameters in order to address the competition between turbulent mixing and reaction, ranging from slow- to fast-reaction rates. We also study the mutual feedback between turbulence evolution driven by the Rayleigh-Taylor instability and front propagation against gravitational acceleration. We quantify both the enhancement of "flame" propagation due to turbulent mixing for the case of slow reactionrate as well as the slowing-down of turbulence growth for the fast-reaction case, when the front quickly burns the gravitationally unstable phase. An increase of intermittency at small scales for temperature characterizes the case of fast reaction, associated to the formation of sharp wrinkled fronts separating pure burnt/unburnt fluids regions.
\end{abstract}

Copyright (c) EPLA, 2011

Introduction. - Many natural and industrial processes involve fluid transport and mixing of passive or active scalar fields; examples include concentration fields of chemicals or biological species as well as the temperature field in natural convection. While many of these phenomena have been the subject of in-depth studies, the cases where chemical reactions are involved, presenting an even richer phenomenology, have received considerably less attention. We address the problem of the interplay of reaction and turbulent mixing in RayleighTaylor (RT) systems (a situation occurring, for example, in thermonuclear burning of type-Ia Supernovae [1-3] or in the inertially confined nuclear fusion [4]) focusing on the different regimes which develop as we vary the

(a) E-mail: ascagliarini@gmail.com ratio between the characteric time scales of underlying turbulence, $\tau_{\text {turb }}$, and the reaction time, $\tau_{R}$. We limit this study to the case of single-step reaction, i.e. two reactant scalar fields are distinguished by a reaction progress variable, proportional to the temperature (see fig. 1). The two different temperatures, in the hot and cold blobs of fluid of our numerical setup, mimick the combustion of a cold mixture of actual reactants into a hot mixture of burnt products [5-7]. The interesting point in this setup is given by the natural competition between gravitational forces, which tends to mix the fluid and to produce a larger and larger mixing layer with uniform temperature, and combustion, which works against this mixing, trying to burn the whole volume and producing a propagating front of given thickness and velocity. Moreover, the global phenomenology is 
Table 1: Parameters for the three sets of runs. Size is $L_{x} \times L_{z}=4096 \times 10000$ for the three runs; Atwood number, At $=$ $\left(T_{d}-T_{u}\right) /\left(T_{d}+T_{u}\right)$; (laminar) Froude number $F r=V_{f}^{2} /\left(g L_{f}\right)$, where $V_{f}$ and $L_{f}$ are the front laminar velocity and thickness (see text), respectively; viscosity $\nu$; thermal diffusivity $\kappa=\nu$; gravity $g$; temperature in the upper half region, $T_{u}$; temperature in the lower half region, $T_{d}$; reaction characteristic time $\tau_{R}$; normalization time, $\tau=\sqrt{L_{x} /(g A t)}$.

\begin{tabular}{|l|cccccccc|}
\hline & $A t$ & $F r$ & $\nu$ & $g$ & $T_{u}$ & $T_{d}$ & $\tau_{R}$ & $\tau$ \\
\hline run A & 0.05 & $7.5 \times 10^{-3}$ & 0.005 & $2.67 \times 10^{-5}$ & 0.95 & 1.05 & $5 \times 10^{3}$ & $5.5 \times 10^{4}$ \\
run B & 0.05 & $2.4 \times 10^{-4}$ & 0.005 & $2.67 \times 10^{-5}$ & 0.95 & 1.05 & $5 \times 10^{4}$ & $5.5 \times 10^{3}$ \\
run C & 0.05 & $7.5 \times 10^{-6}$ & 0.005 & $2.67 \times 10^{-5}$ & 0.95 & 1.05 & $5 \times 10^{5}$ & $5.5 \times 10^{3}$ \\
\hline
\end{tabular}

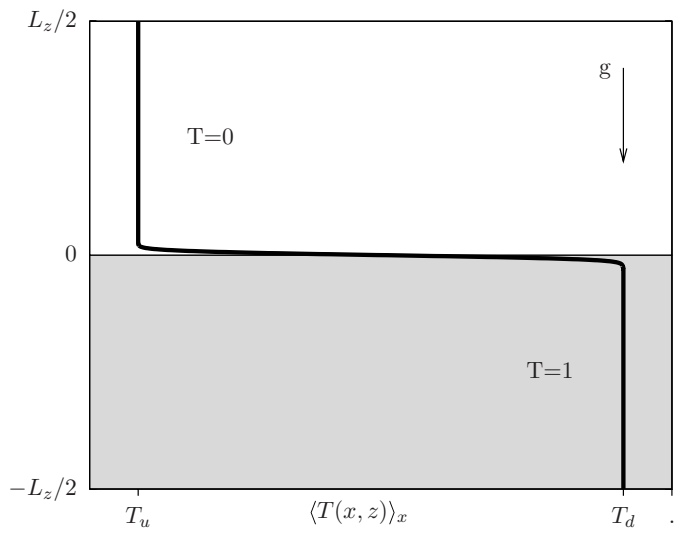

Fig. 1: Initial configuration for the Rayleigh-Taylor system with combustion: cold fluid (fresh fuel) at $T=0$ on top and hot fluid (burnt material) at $T=1$ on bottom. Such temperature jump at the interface is smoothed by a hyperbolic tangent profile with a width of the order of 10 grid points and with a randomly perturbed centre (thus enabling to perform independent runs). The system used is two dimensional and has size $L_{x} \times L_{z}$ plus periodic boundary conditions applied in the streamwise $(x)$ direction. The fluid used is an ideal gas.

complicated by the natural unsteadiness of the underlying RT problem. The Damköhler number, $D a$, is the natural control parameter and is identified by the ratio between the turbulent time scale, $\tau_{\text {turb }}$ and the reaction time scale $\tau_{R}$. Notice that because of RT unsteadiness $D a$ depends on time:

$$
D a(t)=\tau_{\text {turb }}(t) / \tau_{R},
$$

where $\tau_{\text {turb }} \propto t$, as of standard RT phenomenology [7].

We perform highly resolved numerical simulations in 2d, with a resolution up to $4096 \times 10000$ grid points (see table 1 ). The $2 \mathrm{~d}$ setup allowed us to reach a wide scale separation and a time-span large enough to address problems at both small and large Damköhler numbers, something still unfeasible in $3 \mathrm{~d}$. Our study has also a methodological motivation. We adopted a fully consistent thermal lattice Boltzmann method to evolve simultaneously the momentum equations and the advectiondiffusion-reaction equations for temperature. We show here that the method works well also in a non-trivial case where the thermal modes are directly forced by the combustion terms.
The main result of the paper concerns the quantification of the front propagation due to turbulence enhancement for the slow-reaction case, $D a \ll 1$, and the clear signature of a strong feedback on the fluid evolution induced by the front propagation when $D a>1$. In the latter case, we also measure an important increase of the temperature intermittency at small scales.

Equations of motion and numerical setup. - We adopt a numerical scheme based on a recently proposed thermal lattice Boltzmann algorithm [8,9], which is able to reproduce the correct thermohydrodynamics of an ideal gas with good numerical accuracy [10]. To do that, the probability densities $f_{l}(x, t)$ for a particle with velocity $c_{l}$ (belonging to a discrete set, with the index $l$ running over 37 values [9]) at space location $x$ and time $t$ evolve according to the lattice Boltzmann BGK equation [11-13]

$$
f_{l}\left(\boldsymbol{x}+\boldsymbol{c}_{l} \Delta t, t+\Delta t\right)-f_{l}(\boldsymbol{x}, t)=-\frac{\Delta t}{\tau_{L B}}\left(f_{l}-f_{l}^{(e q)}\right)(\boldsymbol{x}, t)
$$

the lhs stands for the free streaming of particles and the rhs represents the relaxation process towards Maxwell equilibrium $f_{l}^{(e q)}(x, t)$ with a characteristic time $\tau_{L B}$ ( $\Delta t$ is the simulation time step). Once the density $(\rho)$, velocity $(u)$ and temperature $(T)$ fields are defined in terms of the lattice Boltzmann populations as

$$
\rho=\sum_{l} f_{l} ; \rho \boldsymbol{u}=\sum_{l} f_{l} \boldsymbol{c}_{l} ; d \rho T=\sum_{l} f_{l}\left|\boldsymbol{c}_{l}-\boldsymbol{u}\right|^{2},
$$

( $d$ is the number of space dimensions), it has been shown $[8,9]$ that the following set of macroscopic equations can be recovered (repeated indexes are summed upon):

$$
\left\{\begin{array}{l}
D_{t} \rho=-\rho \partial_{i} u_{i} \\
\rho D_{t} u_{i}=-\partial_{i} p-\rho g \delta_{i, z}+\nu \Delta u_{i} \\
\rho c_{v} D_{t} T=k \Delta T+\frac{1}{\tau_{R}} R(T)
\end{array}\right.
$$

where $k$ is the thermal conductivity, $\tau_{R}$ the reaction time, $g$ the gravity and $c_{v}=d / 2$ the specific heat at constant volume. Equations (3) are obtained provided that the thermohydrodynamic fields appearing in the equilibrium density functions are properly shifted:

$$
\boldsymbol{u} \rightarrow \boldsymbol{u}+\tau_{L B} \boldsymbol{g} ; T \rightarrow T+\frac{\tau_{L B}\left(\Delta t-\tau_{L B}\right)}{d} g^{2}+\frac{\tau_{L B}}{\tau_{R}} R(T)
$$


The novelty here is in the extra reaction term introduced for the temperature field $[8,9]$.

Concerning the hydrodynamical limit reported in eqs. (3) we observe that extra terms breaking Galilean invariance may emerge at higher order in the ChapmanEnskog expansions [14-18]. In our case such terms can be kept small by controlling the intensity of the external body force. To rule out important sources of errors, we have benchmarked directly the equations of motion in the numerical simulations (see, e.g., fig. 4 of [10]). The energy balance equation has been found very well verified within a few percent for all used resolutions.

Notice also that in the third equation of (3) we have subtracted the compression term $p \nabla \cdot u$ to avoid effects due to a varying heat capacity or global heating of the system coming from a steady increase of the underlying mean pressure ${ }^{1}$.

The reaction rate must be zero in the pure phases, which we set at temperatures $T=1$, for the hot fluid at the bottom, and $T=0$, for the cold fluid on top (see fig. 1), so that $R(0)=R(1)=0$; it must also transforms, irreversibly, the pure cold phase (unstable) into the hot one (stable). A simple model for $R(T)$ with these properties is given by a logistic-type expression

$$
R(T)=T(1-T)
$$

originally proposed $[19,20]$ as a model for the propagation of an advantageous gene in a population and as a model for reactions in a Rayleigh-Taylor system in [7].

The choice of using a lattice Boltzmann algorithm has both physical and computational reasons; from the physical point of view, it allows to tune the degree of compressibility of the flow [9], opening the way to the study of situations where these effects are not negligible at all (e.g., the numerical modelling of nuclear burning in Supernovae explosions [1]). Computationally speaking, the advantage of LB is its very good scaling when run on parallel architectures, due to the fact that the algorithm is completely local in space. Issues on the performance of this code have been studied in [21]. Briefly summarizing, the performance bottleneck is mostly in the streaming routine that accesses memory at sparse addresses and performs a negligible amount of computation. Establishing the appropriate boundary conditions has a negligible impact on performance. Sustained performance is $14-17 \%$ of the peak of the machine (the QPACE Supercomputer [21,22]). Further improvements, mainly merging stream and collision, to avoid unnecessary memory access, may improve the performance up to approximately $20 \%$.

We performed three high-resolution sets of runs (on lattices of $4096 \times 10000$ grid points) with different reaction times (run parameters are collected in table 1). For each set, we carried out several $(\mathcal{O}(10))$ independent runs, in order to enhance statistics.

\footnotetext{
${ }^{1}$ This is done by adding an extra counterterm in the LB shifted temperature inside equilibrium.
}

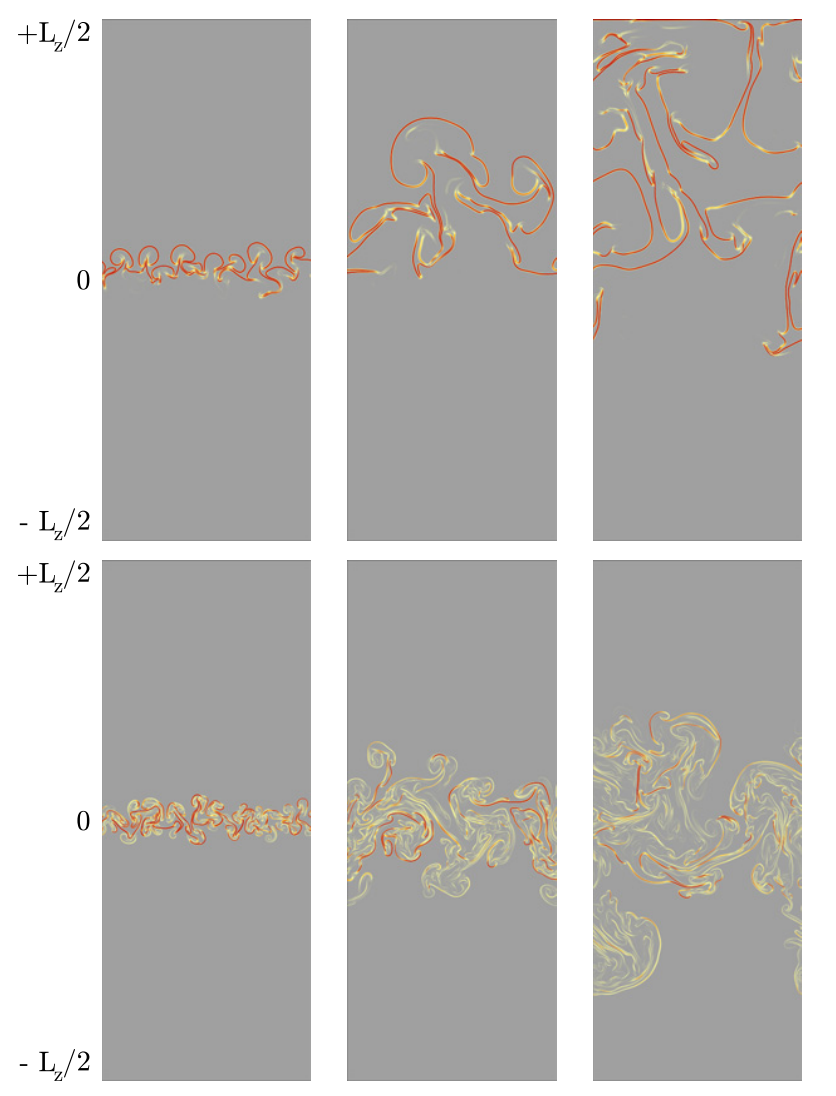

Fig. 2: (Colour on-line) Snapshots of the magnitude field of the temperature gradient for the fast-reaction case, run A (top panel), and for the non-reacting case (bottom panel).

Results and discussion. - Any RT system, even in the case of $\tau_{R} \gg 1$, will eventually reach the fastreaction limit, i.e. a situation where $D a(t) \gg 1$. This is due to the fact that the underlying turbulence slows down adiabatically, $\tau(t) \propto t$. As a consequence, sooner or later the "flame" tends to become active, burning at a rate faster than the turbulence stirring/mixing. Here we study the two regimes $D a \ll 1$ and $D a>1$ and the transition between them.

Mean-temperature-profiles evolution. For large Da, the mixing is effective only at very small scales (where the characteristic times of the fluid motion are shorter), while the reaction tends to make uniform the mixed regions: as a result we get a topology of the temperature field which is made of "patches" separated by rather thin interfaces, which are smoother than the non-reacting RT case [7]; in addition, the front of the hot phase moves, on average, with a non-zero mean drift velocity towards the top. These preliminary features can be better understood, at a pictorial level, looking at fig. 2 , where we show the magnitude of the temperature gradient $|\nabla T|^{2}=\left(\left(\partial_{x} T\right)^{2}+\right.$ $\left.\left(\partial_{z} T\right)^{2}\right)$ at three different times in the evolution for the fastest-reaction rate that we have studied (top panel), and compare it with the non-reacting case (bottom panel). On the other hand, the larger the reaction time $\tau_{R}$ the closer 

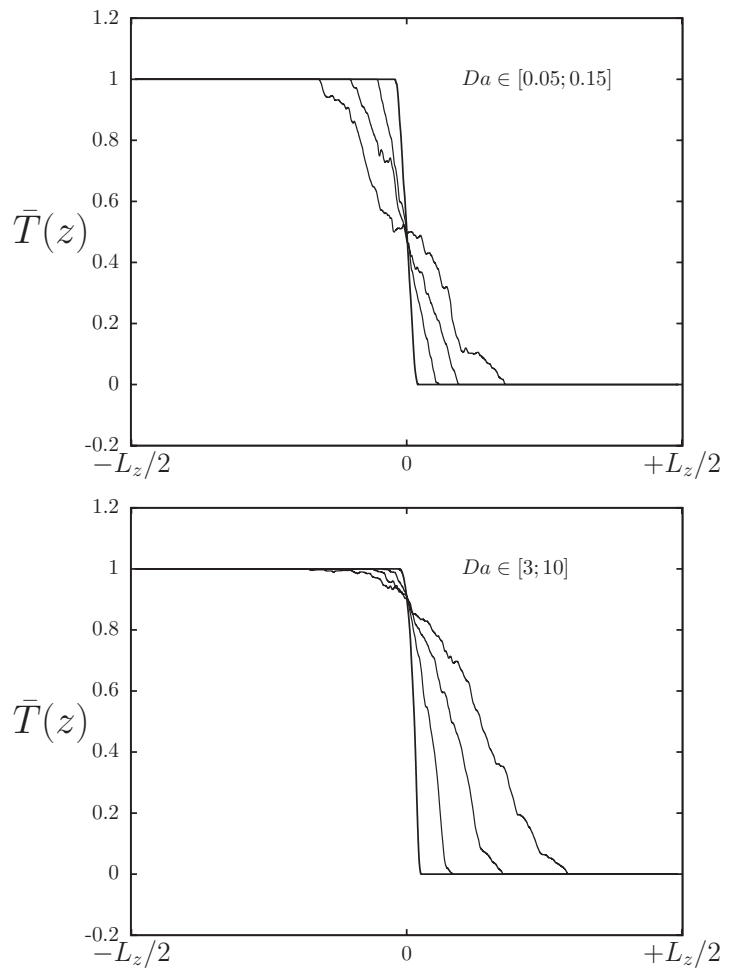

Fig. 3: Mean temperature profiles at various times for run A (bottom panel) and run C (top panel). The latter case is almost identical to the non-reacting case.

is the phenomenology to the standard RT case: to see this we compare in fig. 3 the evolution of the mean temperature profile

$$
\bar{T}(z, t)=\frac{1}{L_{x}} \int T(x, z ; t) \mathrm{d} x
$$

for the two extreme cases in our database, runs A and C: while for $\tau_{R}=5 \times 10^{5}$ the evolution is basically undistinguishable from the usual RT dynamics[9,10], in the fast-reaction case $\left(\tau_{R}=5 \times 10^{3}\right)$ the center of mass of the system clearly moves upwards, due to the burning processes, causing a shift — and an asymmetry - of the mixing region. The propagation of the burnt hot material front against the fresh reactant $(T=0)$ can be quantified by the barycentric coordinate $Z_{f}(t)$, that we define as the following integral $[23,24]$ :

$$
Z_{f}(t)=\int_{-L_{z} / 2}^{+L_{z} / 2} \bar{T}(z, t) \mathrm{d} z
$$

In fig. 4 we plot the function $Z_{f}(t)$ vs. $t$ for the three different reaction rates: the growth of $Z_{f}(t)$ in time is greatly enhanced when going towards smaller $\tau_{R}$.

Front propagation speed. If we integrate the third of eqs. (3) over the whole volume, and divide by $L_{x}$, we get an exact equation for the propagating front speed:

$$
V_{f}(t)=\partial_{t}\left(\int_{-L_{z} / 2}^{+L_{z} / 2} \bar{T}(z, t) \mathrm{d} z\right)=\frac{1}{\tau_{R}}\langle T(1-T)\rangle,
$$

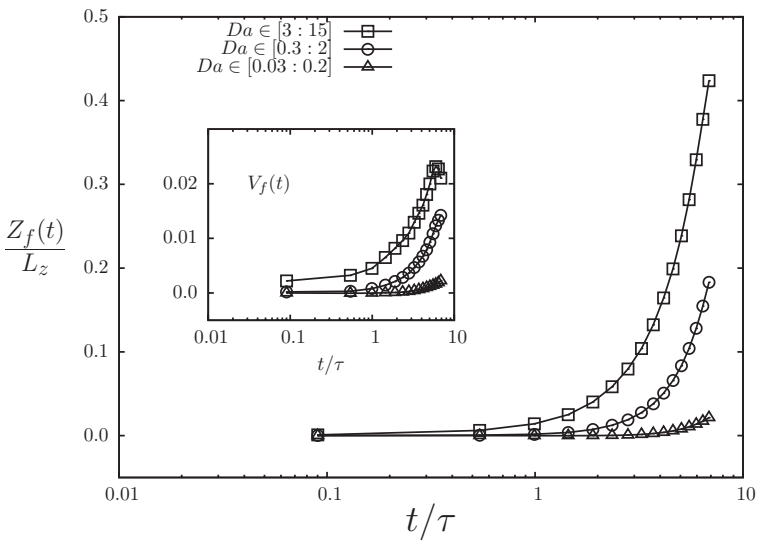

Fig. 4: Reaction front coordinate $Z_{f}$ (normalized by the total vertical box length) and front speed $V_{f}(t)$ (inset) as a function of time for the three sets of runs: the faster the reaction, the more rapidly $Z_{f}$ and $V_{f}$ grow in time.

(where $\left.\langle(\cdot)\rangle=\left(1 / L_{x}\right) \iint(\cdot) \mathrm{d} x \mathrm{~d} z\right)$ since the boundary terms vanish, owing to the periodic conditions on the lateral walls and to the adiabatic condition at top and bottom plates $\left(\left.\nabla T\right|_{z= \pm L_{z} / 2}=0\right)$. For the laminar "flame" the integral can be evaluated exactly (using, for instance, the usual hyperbolic tangent profile) to give an explicit expression for the speed, that is $V_{f} \propto L_{f} / \tau_{R}$, where $L_{f}$ is the front thickness: as the latter can be estimated to be $L_{f} \propto \sqrt{\kappa \tau_{R}}$, we end up with the well-known result:

$$
V_{f} \propto \sqrt{\frac{\kappa}{\tau_{R}}}
$$

that is the front propagates at constant speed.

We now ask what changes when turbulence sets is. In the small $D a$ limit, when turbulence has the time to mix the fluids before reaction becomes active, we are in the so-called pre-mixed combustion. In this case, it has been conjectured $[23,25]$ that the simplest way to extend the result of the laminar case is to replace in expression (8) the molecular diffusivity $\kappa$ with an effective (turbulent) eddy diffusivity $\kappa_{T}$. Let us introduce the mixing layer length as

$$
L(t)=\langle\bar{T}(1-\bar{T})\rangle,
$$

which is directly related to the definition usually used in RT turbulence [26]. A dimensional estimate for the turbulent diffusivity is $\kappa_{T}(t) \sim U(t) L(t)$, where $U$ is a large-scale characteristic velocity (in our case the root mean square velocity). Plugging it into (8), we get

$$
V_{f}(t) \sim \sqrt{\frac{\kappa_{T}}{\tau_{R}}} \sim \sqrt{\frac{U(t) L(t)}{\tau_{R}}} \sim U(t) \sqrt{\frac{(L(t) / U(t))}{\tau_{R}}}
$$

where

$$
V_{f}(t) \sim U(t) \sqrt{\frac{\tau_{\text {turb }}}{\tau_{R}}} \equiv U(t) D a(t)^{1 / 2}
$$


This prediction, probably valid to describe the evolution of slow "flames" in stationary turbulent flows is unlikely to be relevant for RT turbulence. The reason is that in order to observe an "eddy-diffusivity"-driven propagation one needs also a scale separation between the turbulent eddies and the "flame" tickness, something that is not realized by the evolving RT system. On the other hand, we can rewrite (7) exactly as

$$
V_{f}(t)=\frac{1}{\tau_{R}}\left[\langle\bar{T}(1-\bar{T})\rangle-\left\langle\overline{\theta^{2}}\right\rangle\right]
$$

where with $\theta=T-\bar{T}$ we denote the fluctuations with respect to the mean vertical profile. It is clear now that for $D a<1$, the front cannot have any strong influence on the underlying RT evolution and we can identify the first term on the rhs as the mixing layer length $L(t)$. Moreover, we know that in RT temperature fluctuations are almost constant in time and homogeneous inside the mixing layer, so also the second term on the rhs is proportional to the mixing layer extension [27]. A natural prediction for $D a<1$ is therefore

$$
V_{f}(t) \propto \frac{L(t)}{\tau_{R}} ; \quad V_{f}(t) \propto U(t) D a(t) .
$$

Let us notice that this result is valid also for different choices of the reaction term (see also [23]): in fact as long as we may write $R\left(T, \tau_{R}\right)=\left(1 / \tau_{R}\right) f(T)$ (where $f$ is some smooth function of $T$ ), we will have, for the front speed

$$
V_{f}(t)=\frac{1}{\tau_{R}}\langle\overline{f(T)}\rangle ;
$$

moreover, it is always possible to use $\overline{f(T)}=f(\bar{T})+$ fluctuations, and $f$ is non-zero (and positive) only in the mixing layer (being a reaction term). Then, $\langle f(\bar{T})\rangle \propto L(t)$ and the scaling (12) of $V_{f}$ with $D a$ is recovered.

In standard (stationary) turbulent reacting systems, one can check this prediction against experiments/simulations at various $D a$, obtained changing the reaction rate or the underlying turbulent intensity, while in a reacting RT setup we can exploit the fact that $D a=D a(t)$ varies in time. In fig. 5 we plot the front speed (normalized with the root mean square velocity) as a function of $D a$ (which is itself a function of the simulation time) for the three runs. As one can see, our prediction (12) works satisfactorily in a wide range of $D a(t)$, showing deviations only for very small times, where turbulence is not yet developed and the front evolution is strongly influenced by the initial configuration, and for $D a(t)>1$ where it cannot be expected to be valid. In the latter case, data point flatten. We observe the feedback of the "flame" on the turbulent evolution with a sort of synchronization between front propagation and evolution of the turbulent kinetic energy toward a value where $V_{f}(t) \sim U(t)$. Such a behaviour turns out to be in agreement with recent theoretical results obtained through a mean-field approach [28].

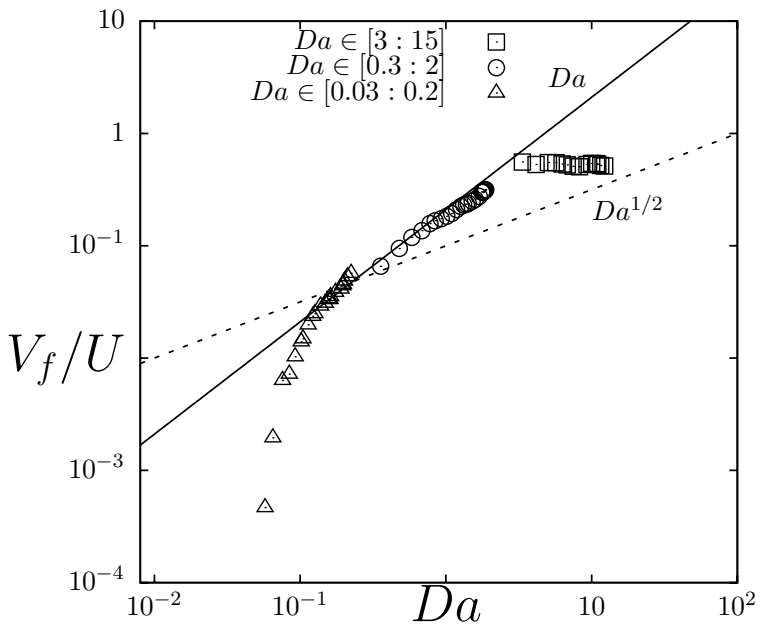

Fig. 5: Front speed normalized by the root mean square (vertical) fluid velocity for the three runs as a function of the Damköhler number $D a(t)$. The solid line represents the theoretically predicted behaviour $V_{f} / U \propto D a$, obtained on the basis that for $D a<1$ "flame" propagates inside the well-mixed mixing layer. The prediction $V_{f} / U \propto D a^{1 / 2}$, obtained from the assumption that in the pre-mixed combustion (slow reaction) regime one can simply substitute the molecular diffusivity with the turbulent one $\kappa \rightarrow \kappa_{T}$ in the expression for the laminar front speed, is also plotted (dashed line).

Small-scale intermittency. When the reaction rate is fast $(D a \gg 1)$, there are no extended regions which are well mixed, since the cold material is rapidly burnt. As a result, the temperature field organizes in patches of pure reactants/products separated by sharp interfaces (being in the so-called "segregated regime"), and, consequently, it has been conjectured that an increased intermittency develops at the small scales [7]. The authors in [7] also derived a phenomenological prediction for the scaling laws of fluid temperature (and velocity) structure functions, according to which, in the asymptotics of $D a \gg 1$, they should follow the relation

$$
S_{T}^{(p)}(R, t) \equiv\left\langle\left|\delta_{R} T\right|^{p}\right\rangle \sim\left(\frac{R}{L(t)}\right)^{2 / 3},
$$

(where $L(t)$ is the mixing layer length), irrespective of the order $p$. From eq. (13) the expression for the flatness reads

$$
F_{T}^{(p)}(R, t)=\frac{\left\langle\left|\delta_{R} T\right|^{p}\right\rangle}{\left\langle\left|\delta_{R} T\right|^{p / 2}\right\rangle^{2}} \sim R^{-2 / 3} L(t)^{2 / 3}
$$

and so it increases with decreasing $R$ for all orders, a clear indication of strong small-scales intermittency. In fig. 6 we show the growth of $F_{T}^{(4)}(R=1, t)$ as a function of $L(t)$, for the three runs: the flatness for run A, corresponding to the smallest reaction time, is in good agreement, within error bars, with the prediction of eq. (14), $F_{T}^{(4)} \sim L^{2 / 3}$; instead, at increasing $\tau_{R}$, intermittency is depleted and the flatness grows more slowly, at a 


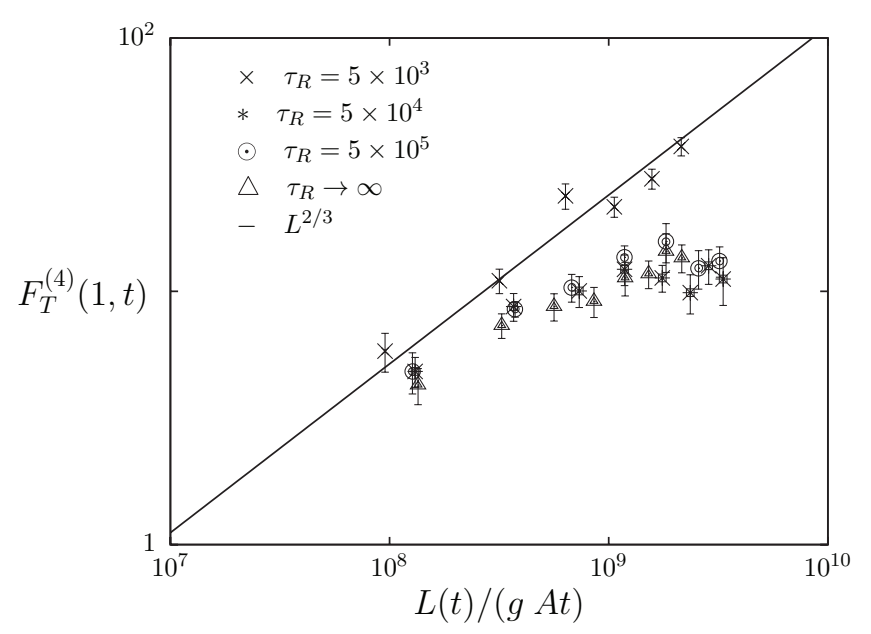

Fig. 6: The 4-th order flatness $F_{T}^{(4)}(R=1, t)$ for the three runs and for the non-reacting $\mathrm{RT}\left(\tau_{R} \rightarrow \infty\right)$. Data from run A $(D a \gg 1)$ agree well, within error bars, with the prediction given by eq. (14), $F_{T}^{(4)} \sim L^{2 / 3}$.

rate comparable (within error bars) with the non-reacting RT case, whose data are also reported for comparison.

Conclusions. - We used a self-consistent thermal lattice Boltzmann algorithm to perform numerical simulations of $2 \mathrm{~d}$ Rayleigh-Taylor turbulence, in the presence of chemical reactions between hot and cold fluids. The reaction was modelled by means of a Fisher-KolmogorovPetrovsky-Piskunov source term in the temperature equation; this term has been introduced by a suitable shift of the temperature field appearing in the equilibria of the lattice Boltzmann equation.

We analyzed the crossover among the various regimes emerging from the competition of turbulent mixing and reaction, going from the segregated $\left(\tau_{R} \gg \tau_{\text {turb }}\right)$ to the well-mixed one $\left(\tau_{R} \ll \tau_{\text {turb }}\right)$. We showed that, in the latter case, the effect of turbulence is to enhance the reaction front speed leading to an homogeneous burning in the whole mixing layer region. On the other hand, for moderate and large Damköhler, there is a feedback of the reaction on the statistical properties of the temperature field, resulting in increased intermittency at small scales in reasonable accordance with the prediction of [7].

$$
* * *
$$

We thank A. Celani and M. Cencini for useful suggestions and the QPACE development team for support during the implementation of our code and execution of the runs. We acknowledge access to the QPACE and eQPACE systems. We acknowledge support from CASPUR HPC-grant 2010.

\section{REFERENCES}

[1] Zingale M., Woolsey S. E., Rendleman C. A., Day M. S. and Bell J. B., Astrophys. J., 1021 (2005) 632.

[2] Khokhlov A. M., Astrophys. J., 449 (1995) 695.

[3] Gamezo V. N., Khokhlov A. M., Oran E. S., Chtchelkanova A. Y. and Rosenber R. O., Science, 299 (2003) 77.

[4] Freeman J. R., Clauser M. J. and Thompson S. L., Nucl. Fusion, 223 (1997) 17.

[5] Cetegen B. M. and Kasper K. D., Phys. Fluids, 8 (1996) 2974.

[6] Tieszen S. R., Annu. Rev. Fluid Mech., 33 (2001) 67.

[7] Chertkov M., Lebedev V. and Vladimirova N., J. Fluid Mech., 633 (2009) 1.

[8] Sbragaglia M., Benzi R., Biferale L., Chen H., Shan X. and Succi S., J. Fluid Mech., 628 (2009) 299.

[9] Scagliarini A., Biferale L., Sbragaglia M., Sugiyama K. and Toschi F., Phys. Fluids, 22 (2010) 055101.

[10] Biferale L., Mantovani F., Sbragaglia M., Scagliarini A., Toschi F. and Tripiccione R., Phys. Fluids, 22 (2010) 115112.

[11] Bathnagar P.-L., Gross E. and Krook M., Phys. Rev., 94 (1954) 511.

[12] Wolf-Gladrow D., Lattice-gas Cellular Automata and Lattice Boltzmann Models (Springer, New York) 2000.

[13] Succi S., The Lattice Boltzmann Equation and its Applications (Oxford Science publications) 2001.

[14] Holdych D. J., Rovas D., Georgiadis J. G. and Buchius R. O., Int. J. Mod. Phys. C, 9 (1998) 1393.

[15] Inamuro T., Konishi N. and Ogino F., Comput. Phys. Commun., 129 (2000) 32.

[16] Kalarakis A. N., Burganos V. N. and Payatakes A. C., Phys. Rev. E, 65 (2002) 056702.

[17] Wagner A. J. and Li Q., Physica A, 362 (2006) 105.

[18] Lallemand P. and Luo L.-S., Phys. Rev. E, 61 (2000) 6546.

[19] Fisher R., Ann. Eugen., 7 (1937) 355.

[20] Kolmogorov A. N., Petrovsky I. G. and Piskunov N. G., Bull. Moskovskogo Gosudartsvennogo Univ. Mat. Mekh., 1 (1937) 1.

[21] Biferale L., Mantovani F., Pivanti M., Sbragaglia M., Scagliarini A., Schifano S. F., Toschi F. and Tripiccione R., Procedia Comput. Sci., 1 (2010) 1069.

[22] Goldrian G. et al., Comput. Sci. Eng., 10 (2008) 46.

[23] Koudella C. R. and Neufeld Z., Phys. Rev. E, 70 (2004) 026307.

[24] Constantin P., Kiselev A., Oberman A. and Rhyzik L., Arch. Ration. Mech. Anal., 154 (2000) 53.

[25] Damköhler G., Z. Elektrochem. Angew. Phys. Chem., 46 (1940) 601.

[26] Cавот W. H. and Cook A. W., Nat. Phys., 2 (2006) 562.

[27] Chertkov M., Phys. Rev. Lett., 91 (2003) 115001.

[28] Brandenburg A., Erland L., Haugen N. and Babkovskaia N., Phys. Rev. E, 83 (2011) 016304. 